\title{
Neoadjuvant treatment with tyrosine kinase inhibitors in patients with resectable non-small cell lung cancer
}

\author{
Fernando Franco, Mariano Provencio \\ Medical Oncology Department, Puerta de Hierro Hospital, Madrid, Spain \\ Correspondence to: Fernando Franco. Medical Oncology Department, Puerta de Hierro Hospital, Manuel de Falla Street \#1, 28222 Majadahonda, \\ Madrid, Spain. Email: f3franc@gmail.com. \\ Provenance: This is an invited article commissioned by the Editorial Office, Fournal of Thoracic Disease. \\ Comment on: Zhang Z, Lin J, Peng S, et al. Radical surgical resection after neoadjuvant targeted therapy in non-small cell lung cancer: a single-center \\ retrospective study of 6 cases. J Thorac Dis 2019;11:248-51.
}

Submitted Sep 15, 2019. Accepted for publication Sep 23, 2019.

doi: $10.21037 /$ jtd.2019.09.68

View this article at: http://dx.doi.org/10.21037/jtd.2019.09.68

We have carefully read the article written by Dr. Zhang et al. in which they reported your surgical experience in six patients with resectable epidermal growth factor receptor (EGFR) mutated non-small cell lung cancer (NSCLC) after gefitinib as neoadjuvant therapy (1). We know that the stage III of NSCLC comprises an important heterogeneous group of patients with big differences in terms of location and extension of the primary tumour as well as the mediastinal lymph nodes metastasis. The current tumornode-metastasis (TNM) $\left(8^{\text {th }}\right.$ edition) shows us better the differences of this disease in terms of survival according to the stage of the disease at the moment of diagnosis (2). Currently, there are different therapeutic strategies used in the treatment of these tumours, including surgery followed by adjuvant chemotherapy (CT), induction CT and/or radiotherapy. For patients with unresectable disease, the indicated treatment is the concurrent chemoradiotherapy followed by durvalumab without any progression of the disease. The benefit of durvalumab was confirmed in the PACIFIC trial in which the overall survival (OS) was significantly longer in the group of treatment compared with the placebo group [hazard ratio (HR) for death, 0.68; 95\% confidence interval (CI), 0.470-0.997; $\mathrm{P}=0.0025]$. The improvement in progression-free survival (PFS) was demonstrated also with a median of $17.2 v s .5 .6$ months in the intervention group (HR, 0.51; 95\% CI, 0.41-0.63) $(3,4)$. This is the reason why it is very important to discuss each case and select the best treatment.

In several studies have been demonstrated the improvement in survival of the patients treated with platinum-based adjuvant $\mathrm{CT}$ after a completely resected NSCLC. These results were confirmed in the LACE metaanalysis which included more than 4,500 patients and showed a decrease in the risk of death of $5.4 \%$ at 5 -year in patients treated with CT compared to the control group (HR, 0.89). However, it is necessary to remember that this benefit only was statistically significant in patients with stage II and IIIA of the disease (HR, 0.83) (5). The induction therapy is an other strategy evaluated also in this group of patients but the data is limited, compared with the adjuvant CT. However, comparative analyses seem to indicate that there are no differences in OS, although it can improve the results of surgery $(6,7)$. The EGFR tyrosine kinase inhibitors (TKIs) has been evaluated also in induction therapy in patients potentially resectable of NSCLC, both in a molecularly selected population or not (8-10). In all cases the neoadjuvant therapy with TKIs has demonstrated good tolerability and safety but the results in terms of objective response rate (ORR) and survival are unequal.

In his article, Dr. Zhang presents us a total of six cases of patients operated for primary lung adenocarcinoma after a period of 8 weeks of neoadjuvant treatment with gefitinib. Five of the patients had a stage IIIA of the disease and one case had a stage IIIB, according to the $7^{\text {th }}$ edition of the TNM.

However, we know that the stage IIIA includes a highly heterogeneous group of the disease and the complete 
staging of the 6 cases was not described. For us, this is an important data to understand the impact of treatment on the down-staging of the tumour. In all cases, the status of EGFR was analysed previously to start the TKI therapy ( 3 cases of exon 19 deletion and 3 cases of exon 21 L858R mutation). The treatment tolerance, the type of surgery (lobectomy and mediastinal lymphadenectomy) and the postoperative pathologic staging have been described in only one isolated case.

We consider that despite being a series with a small number of cases, the scientific value is important and we want to contrast its results with the scientific evidence published so far. The CSLC-0702 (NCT00600587) is the first phase II study of biomarker-guided neoadjuvant treatment for patients with resectable NSCLC (stage IIIA-N2 confirmed) based on EGFR mutation status (8). In this clinical trial the patients were randomized according to the status of EGFR to receive neoadjuvant CT for three cycles (gemcitabine plus carboplatin) or erlotinib during 42 days before the surgery. The primary endpoint was the ORR and the secondary were the PFS and the OS. The total number of patients included was 42 (twelve in every arm). The results show us an ORR of $41.7 \%$ in all population, being of $58.3 \%(7 / 12)$ in the EGFR mutant group (treated with TKI) versus $25 \%$ in the $\mathrm{CT}$ arm $(\mathrm{P}=0.18)$. The PFS was longer in the CT group (9 months; $95 \%$ CI, $3.8-19.0$ months) compared with the TKI group (6.9 months; $95 \%$ CI, 3.1-15.0 months), there were no statistically significant differences $(\mathrm{P}=0.07)$. The $\mathrm{OS}$ was superior in the CT group with a median of 28.1 (95\% CI, 0-66) vs. 14.5 (95\% CI, 1-28) months for the erlotinib $(\mathrm{P}=0.201)$. In general, both treatments were well tolerated and any unexpected toxicities were observed. These results allow us to see that the neoadjuvant treatment with erlotinib setting tended to show an improved response but without any benefit in the PFS or in the OS. However, the small number of patients included in this trial can justify the absence of differences of efficacy between the treatment groups.

Another study, that has recently evaluated the role of neoadjuvant erlotinib in patients with stage IIIA-N2 of EGFR mutated NSCLC, was conducted in a single institution in China (Shanghai Chest Hospital), NCT01217619 (11). This is a prospective phase II study with a single arm, in which the patients received erlotinib $150 \mathrm{mg}$ per day for 56 days in the preoperative period. The primary endpoint was the radical resection rate and the secondary one included ORR, PFS, OS, complete pathologic response (CPR) rate, quality of life (QoL) and biomarkers. Nineteen EGFR mutated patients were enrolled and treated, $63 \%$ with an exon 19 deletion and $47 \%$ with an exon 21 L858R mutation. After finishing the neoadjuvant treatment, 14 patients underwent surgery and the remaining 5 patients did not: 2 of them had progression of the disease, 2 more had severe adverse events and 1 patient refused the intervention. The radical resection rate was $68.4 \%$ with a $21 \%$ rate of pathological downstaging, without any CPR. The ORR was $42.1 \%$ with an average of 10.3 months of PFS in the group of the operated patients. Among all 19 patients who received neoadjuvant erlotinib, the median of PFS was 11.2 months and the OS was 51.6 months. In 8 of the operated patients the nextgeneration sequencing revealed that 7 of them had a TP53 gene mutation associated to an EGFR mutation. In these cases, the PFS was shorter ( 8 months) than the one in patients without this alteration. The authors concluded that the neoadjuvant erlotinib is a well-tolerated treatment and it improved the radical resection rate in this population. Finally, we want to comment the EMERGING-CTONG 1103 study which is a multicenter, phase II, randomized controlled trial of erlotinib versus gemcitabine plus cisplatin as neoadjuvant/adjuvant therapy in patients with stage IIIA-N2 of NSCLC with EGFR mutations in exon 19 or 21 . In the TKI arm, the patients received erlotinib $150 \mathrm{mg}$ per day as neoadjuvant therapy during 42 days and adjuvant therapy with the same dose up to 12 months, while in the CT arm were administrated 2 cycles of neoadjuvant CT and 2 more cycles after the surgical intervention. The primary endpoint was the ORR and the secondary included CPR rate, PFS, OS, safety, and tolerability. Seventy-two patients were randomized (386 screened) and 71 were included in the safety analysis. The ORR for neoadjuvant treatment was $54.1 \%$ in the erlotinib arm compared with the $34.3 \%$ in the CT group (odds ratio, 2.26; 95\% CI, 0.87-5.84; $\mathrm{P}=0.092$ ), without any CPR in either arm. The lymph node downstaging was low in both groups, but higher in the erlotinib arm $(10.8 \%$ vs. $2.9 \%)$. Only 3 patients achieved a major pathological response in the TKI group and none in the control group. The median of PFS was significantly longer in patients treated with erlotinib (21.5 months) compared with the CT arm (11.4 months), the HR was 0.39 (95\% CI, 0.23-0.67; $\mathrm{P}=0.001)$. The median of the follow-up was 32.5 months and no differences were observed in the OS between the two arms, 45.8 vs. 39.2 months in the TKI and CT group respectively (HR, 0.77; 95\% CI, 0.41-1.45; $\mathrm{P}=0.417$ ). Although the primary objective was not achieved, this study has been demonstrated a significantly improved in PFS in 
patients treated with neoadjuvant/adjuvant erlotinib (12).

In the lasts years the impact of immunotherapy (IO) has been demonstrated in patients with advanced NSCLC, both in the first-line of treatment and in later lines (13-16). However, the anti-tumoral activity and the clinical benefit of IO in patients with EGFR mutated advanced NSCLC is lower than in wild-type population. Only the IMPOWER 150 study has demonstrated that the addition of IO to the scheme of CT (carboplatin/paclitaxel) plus bevacizumab improved the PFS and the ORR in EGFR mutated patients (17). Currently, the use of IO in monotherapy or combinated with CT is being evaluated in several clinical trials as a new induction treatment in patients with potentially resectable disease. In May 2018, Forde $e t$ al. published a study that evaluates the use of neoadjuvant nivolumab at a dose of $3 \mathrm{mg} / \mathrm{kg}$ every 2 weeks in a small cohort of patients with stages I-IIIA of NSCLC (NCT02259621) (18). From all 21 patients included (without any information about the status of EGFR), 20 were subsequently operated. The major pathologic response (MPR) rate was $45 \%$ and the treatment was well tolerated. In the annual meeting of ASCO 2019, were presented de partial results of NADIM study (NCT03081689) which is a phase II, single-arm and openlabel multicenter study for patients with resectable stage IIIA-N2 NSCLC treated with the neoadjuvant combination of CT (carboplatin/paclitaxel) plus nivolumab every three weeks for 3 cycles. The primary endpoint in this study was the PFS at 24 months and the data of efficacy is explored using objective pathologic response criteria. With 30 patients included and 13 underwent surgery, the tolerability of the treatment was excellent without any severe adverse events. The CPR rate was $69.2 \%$ (95\% CI, 38.6-90.9\%) plus 2 cases with a MPR, defined as $<10 \%$ viable tumour cells in the resection specimen. Considering both types of response (CPR and MPR), the ORR was $84.6 \%$ (95\% CI, 54.6-98.1\%). These results are very important in the development of new therapeutic strategies in the management of the patients with locally advanced NSCLC (19). The update data of this study with 46 patients included, presented in IASLC 2019 in Barcelona, confirmed the high rate of objective pathological responses in these patients. Only one case has been reported with a known EGFR mutation that reached a higher response but subsequently relapsed in the central nervous system. Another study, similar to the previous one, but with fewer patients (14 cases) and using 4 cycles of neoadjuvant CT (carboplatin/nab-paclitaxel) plus atezolizumab, showed important pathological responses, but more discrete than the NADIM study (20).

With all these results, we consider that it is important to continue the research in order to clarify the impact of neoadjuvant therapy with TKI in EGFR mutated locally advanced lung cancer. Despite the good results of the use of TKIs in the first-line of treatment, they can not be extrapolated to preoperative management of these tumours without having more consistent data. There are serious doubts about the ideal period of time necessary for neoadjuvant treatment with TKIs and whether this should be followed by adjuvant therapy or not. It is necessary that new studies will be designed to evaluate this therapeutic strategy, including more extensive molecular data (sensitizing and resistance mutations, common and uncommon mutations, coexistence of diverse mutations and the tumour mutational burden). Another question which arises is whether the benefit of the neoadjuvant treatment, combining CT plus IO, can have the same impact in EGFR mutated and wild-type patients.

\section{Acknowledgments}

None.

\section{Footnote}

Conflicts of Interest: The authors have no conflicts of interest to declare.

Ethical Statement: The authors are accountable for all aspects of the work in ensuring that questions related to the accuracy or integrity of any part of the work are appropriately investigated and resolved.

\section{References}

1. Zhang Z, Lin J, Peng S, et al. Radical surgical resection after neoadjuvant targeted therapy in non-small cell lung cancer: a single-center retrospective study of 6 cases. J Thorac Dis 2019;11:248-51.

2. Amin MB, Greene FL, Edge SB, et al. The eighth edition AJCC cancer staging manual: continuing to build a bridge from a population-based to a more "personalized" approach to cancer staging. CA Cancer J Clin 2017;67:93-9.

3. Antonia SJ, Villegas A, Daniel D, et al. Durvalumab after chemoradiotherapy in stage III non-small-cell lung cancer. 
N Engl J Med 2017;377:1919-29.

4. Antonia SJ, Villegas A, Daniel D, et al. Overall survival with durvalumab after chemoradiotherapy in stage III NSCLC. N Engl J Med 2018;379:2342-50.

5. Pignon JP, Tribodet H, Scagliotti GV, et al. Lung adjuvant cisplatin evaluation: a pooled analysis by the LACE collaborative group. J Clin Oncol 2008;26:3552-9.

6. Lim E, Harris G, Patel A, et al. Preoperative versus postoperative chemotherapy in patients with resectable non-small cell lung cancer: systematic review and indirect comparison meta-analysis of randomized trials. J Thorac Oncol 2009;4:1380-8.

7. NSCLC Meta-analysis Collaborative Group. Preoperative chemotherapy for non-small-cell lung cancer: a systematic review and meta-analysis of individual participant data. Lancet 2014;383:1561-71.

8. Zhong W, Yang X, Yan H, et al. Phase II study of biomarker-guided neoadjuvant treatment strategy for IIIA-N2 non-small cell lung cancer based on epidermal growth factor receptor mutation status. J Hematol Oncol 2015;8:54.

9. Schaake EE, Kappers I, Codrington HE, et al. Tumor response and toxicity of neoadjuvant erlotinib in patients with early-stage non-small-cell lung cancer. J Clin Oncol 2012;30:2731-8.

10. Han B, Xiong L, Sun J, et al. Erlotinib as neoadjuvant treatment in endobronchial ultrasound confirmed stage IIIA-N2 non-small cell lung cancer (NSCLC) patients with epidermal growth factor receptor (EGFR) mutation (exon 19 or 21) (NCT01217619, ESTERN): a prospective, single arm, phase II clinical trial. Ann Oncol 2015;26:i24.

11. Xiong L, Li R, Sun J, et al. Erlotinib as neoadjuvant therapy in stage IIIA (N2) EGFR mutation-positive nonsmall cell lung cancer: a prospective, single-arm, phase II study. Oncologist 2019;24:157-e64.

12. Zhong WZ, Chen KN, Chen C, et al. Erlotinib versus gemcitabine plus cisplatin as neoadjuvant treatment of

Cite this article as: Franco F, Provencio M. Neoadjuvant treatment with tyrosine kinase inhibitors in patients with resectable non-small cell lung cancer. J Thorac Dis 2019;11(10):4092-4095. doi: 10.21037/jtd.2019.09.68 stage IIIA-N2 EGFR-mutant non-small-cell lung cancer (EMERGING-CTONG 1103): a randomized phase II study. J Clin Oncol 2019;37:2235-45.

13. Brahmer J, Reckamp KL, Baas P, et al. Nivolumab versus docetaxel in advanced squamous-cell non-small-cell lung cancer. N Engl J Med 2015;373:123-35.

14. Reck M, Rodríguez-Abreu D, Robinson AG, et al. Pembrolizumab versus chemotherapy for PD-L1positive non-small-cell lung cancer. $\mathrm{N}$ Engl J Med 2016;375:1823-33.

15. Rittmeyer A, Barlesi F, Waterkamp D, et al. Atezolizumab versus docetaxel in patients with previously treated non-small-cell lung cancer (OAK): a phase 3, openlabel, multicentre randomised controlled trial. Lancet 2017;389:255-65.

16. Langer CJ, Gadgeel SM, Borghaei H, et al. Carboplatin and pemetrexed with or without pembrolizumab for advanced, non-squamous non-small-cell lung cancer: a randomised, phase 2 cohort of the open-label KEYNOTE-021 study. Lancet Oncol 2016;17:1497-508.

17. Reck M, Mok TSK, Nishio M, et al. Atezolizumab plus bevacizumab and chemotherapy in non-small-cell lung cancer (IMpower150): key subgroup analyses of patients with EGFR mutations or baseline liver metastases in a randomised, open-label phase 3 trial. Lancet Respir Med 2019;7:387-401.

18. Forde PM, Chaft JE, Smith KN, et al. Neoadjuvant PD-1 blockade in resectable lung cancer. $\mathrm{N}$ Engl J Med 2018;378:1976-86.

19. Provencio-Pulla M, Nadal-Alforja E, Cobo M, et al. Neoadjuvant chemo/immunotherapy for the treatment of stages IIIA resectable non-small cell lung cancer (NSCLC): a phase II multicenter exploratory study-NADIM studySLCG. J Clin Oncol 2018;36:8521.

20. Shu CA, Grigg C, Chiuzan C, et al. Neoadjuvant atezolizumab + chemotherapy in resectable non-small cell lung cancer (NSCLC). J Clin Oncol 2018;36:abstr 8532. 